\title{
The electrorheology of barium titanate suspensions
}

\author{
Peter J. Rankin and Daniel J. Klingenberg ${ }^{\text {a) }}$ \\ Department of Chemical Engineering and Rheology Research Center, \\ University of Wisconsin, Madison, Wisconsin 53706
}

(Received 23 October 1997; final revision received 10 February 1998)

\begin{abstract}
Synopsis
Barium titanate/insulating oil suspensions were investigated to determine the dielectric polarization mechanisms that govern their electrorheological (ER) behavior. The dynamic yield stress of 19.3 vol \% suspensions exhibited Maxwell-Wagner-like frequency dependence, with the dynamic yield stress increasing significantly with ac electric field frequency, as expected for suspensions composed of particles with large dielectric constants. The dynamic yield stress at a given frequency was proportional to $E^{m}$, where $E$ is the applied electric field strength and the exponent $m$ increased with frequency. For electric field strengths of $2 \mathrm{kV} / \mathrm{mm}$ at $1 \mathrm{kHz}$, dynamic yield stresses were approximately $500 \mathrm{~Pa}$. Rheological experiments in which particle surface and oil conductivities were varied suggest that the dielectric relaxation of these suspensions is controlled by the particles' bulk conductivity. The dielectric relaxation depended strongly on field strength, becoming broader with increasing field strength. Harmonic analysis of the current passing through the suspensions verified that the nonlinear contribution to the apparent suspension conductivity increased with $E$ and decreased with frequency. The current harmonics for barium titanate/dry air suspensions were similar to those of the oil based suspensions, suggesting that nonlinear conduction may arise from field-enhanced dissociation of surface groups, as opposed to field-enhanced dissociation of ion pairs within the continuous phase. (C) 1998 The Society of Rheology. [S0148-6055(98)01403-5]
\end{abstract}

\section{INTRODUCTION}

Rheological properties of electrorheological (ER) fluids, which consist of weakly conducting particles in weakly conducting liquids, are dramatically altered by applied electric fields. The steady-state rheological response in shearing flows is commonly modeled as a Bingham fluid, with an electric field-dependent dynamic yield stress, $\tau_{o}\left(E_{0}\right)$,

$$
\begin{gathered}
\tau\left(\dot{\gamma}, E_{0}\right)=\tau_{o}\left(E_{0}\right)+\eta_{\mathrm{p} 1} \dot{\gamma} \text { for } \tau>\tau_{o}, \\
\dot{\gamma}=0 \text { for } \tau<\tau_{o},
\end{gathered}
$$

where $\tau$ is the shear stress, $E_{0}$ is the applied field strength, $\eta_{\mathrm{pl}}$ is the plastic viscosity, and $\dot{\gamma}$ is the shear rate. Winslow (1949) first reported the ER effect, and it has been reviewed in numerous publications [Deinega and Vinogradov (1984); Block and Kelly (1988); Gast and Zukoski (1989); Jordan and Shaw (1989); Weiss et al. (1993); Parthasarathy and Klingenberg (1996); and references therein]. Many devices can benefit from the ability to electronically control stress transfer, including active shock absorbers and engine mounts, clutches, brakes, alternators, power steering pumps, control valves, and

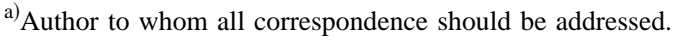


artificial joints [Hartsock et al. (1991)]. Despite potential applications, there are currently no commercially available ER devices, due largely to a lack of suitable fluids. ER fluids must be noncorrosive, active over a wide temperature range, stable against settling, irreversible flocculation, and chemical degradation, and draw limited power. In this paper, we report the electrorheological response of barium titanate/low permittivity insulating oil suspensions. As expected for suspensions containing particles with large dielectric constants (dielectric constant of barium titanate $\approx 2000$ ) [Ginder and Ceccio (1995)], these suspensions exhibit large yield stresses for sufficiently large field frequenciesyield stresses for $19.3 \mathrm{vol} \%$ suspensions are approximately $500 \mathrm{~Pa}$ at $2 \mathrm{kV} / \mathrm{mm}$ and 1 $\mathrm{kHz}$.

A primary limitation to the development of suitable ER fluids is an incomplete understanding of the underlying mechanisms. A number of theories have been proposed to explain the ER effect, but the electrostatic polarization (EP) model appears to qualitatively explain most features [Winslow (1949)]. The model attributes the ER effect to the field-induced polarization of the dispersed phase relative to the continuous phase. A particle's charge distribution arising from this polarization can be described to leading order as a dipole. The dipolar particles align head-to-tail in the direction of the electric field, resulting in the experimentally observed fibrous structure. These fibrous columns must be broken for the suspension to flow; the yield stress is proportional to the force required to break these columns.

It is clear that the magnitude of the ER response depends on the details of the particle polarization, which controls the electrostatic interparticle force. Determining this force requires solving for the electrostatic potential field within the suspension. The idealized electrostatic polarization (IEP) model treats this problem in the simplest manner, ignoring both dispersed and continuous phase electrical conductivities and attributing particle polarization exclusively to permittivity differences. Although this approach captures many prominent features of the ER effect (e.g., $\tau_{o} \propto E_{o}^{2}$ ), it fails to describe other important experimental observations, such as the dependence of rheological properties on electric field frequency.

For dc and low-frequency ac electric fields, particle polarization is controlled by conductivity, rather than permittivity differences [Anderson (1992); Davis (1992a); Davis (1992b)]. The Maxwell-Wagner model describes particle polarization arising from both permittivity and conductivity differences [see, for example, Parthasarathy and Klingenberg (1996), and references therein]. In the point-dipole limit, the time-averaged force on a particle at the origin due to a second particle at $(r, \theta)$ in a uniform electric field, $\mathbf{E}_{\mathbf{0}}$ $=\operatorname{Re}\left(E_{0} e^{i \omega t}\right) \mathbf{e}_{z}$, is

$$
F=\frac{3}{4} \pi \epsilon_{0} \epsilon_{c} a^{2}\left(\frac{2 a}{r}\right)^{4} \beta_{\mathrm{eff}}^{2} E_{\mathrm{rms}}^{2}\left[\left(3 \cos ^{2} \theta-1\right) \mathbf{e}_{r}+\sin 2 \theta \mathbf{e}_{\theta}\right],
$$

where $\omega$ is the ac field frequency, $\mathbf{e}_{i}$ is the unit vector in the $i$ direction, $\epsilon_{0}$ is the permittivity of free space, $a$ is the particle radius, $E_{\mathrm{rms}}=E_{0} / \sqrt{2}$, and $\beta_{\text {eff }}$ is the "effective relative polarizability,"

$$
\beta_{\mathrm{eff}}^{2}(\omega)=\frac{\beta_{c}^{2}+\beta_{d}^{2}\left(\omega t_{m w}\right)^{2}}{1+\left(\omega t_{m w}\right)^{2}}
$$

where 


$$
\beta_{d}=\frac{\epsilon_{p}-\epsilon_{c}}{\epsilon_{p}+2 \epsilon_{c}}, \quad \beta_{c}=\frac{\sigma_{p}-\sigma_{c}}{\sigma_{p}+2 \sigma_{c}}, \quad t_{m w}=\epsilon_{0} \frac{\epsilon_{p}+2 \epsilon_{c}}{\sigma_{p}+2 \sigma_{c}} .
$$

$\epsilon_{p}$ and $\epsilon_{c}$ are the dispersed and continuous phase relative dielectric constants, respectively, and $\sigma_{p}$ and $\sigma_{c}$ are the respective electrical conductivities. $\beta_{d}$ depends on the ratio of dispersed to continuous phase permittivities, and the larger the ratio the closer $\beta_{d}$ is to 1. In the same manner, $\beta_{c}$ depends on the ratio of dispersed to continuous phase conductivities. If particles exhibit surface conductivity, $\beta_{c}$ and $t_{m w}$ are modified. The surface conductivity, $\lambda^{s}$, and the bulk conductivity, $\sigma_{p}$, contribute to an effective particle conductivity [O’Konski (1960)],

$$
\sigma_{p}^{\mathrm{eff}}=\sigma_{p}+\frac{2 \lambda^{s}}{a}
$$

leading to

$$
\beta_{c}=\frac{\sigma_{p}+\frac{2 \lambda^{s}}{a}-\sigma_{c}}{\sigma_{p}+\frac{2 \lambda^{s}}{a}+2 \sigma_{c}}, \quad t_{m w}=\epsilon_{0} \frac{\epsilon_{p}+2 \epsilon_{c}}{\sigma_{p}+\frac{2 \lambda^{s}}{a}+2 \sigma_{c}} .
$$

Since the dynamic yield stress is directly proportional to the interparticle force, the Maxwell-Wagner model predicts that $\tau_{o} \alpha \beta_{\mathrm{eff}}^{2} E_{\mathrm{rms}}^{2}$, where the frequency dependence is determined by the suspension dielectric properties through $\beta_{\mathrm{eff}}(\omega)$. $\beta_{\mathrm{eff}}^{2}$ as a function of frequency for different relative magnitudes of $\beta_{d}$ to $\beta_{c}$ is illustrated in Fig. 1 . In the limit of large frequencies, the dynamic yield stress is determined by permittivity differences,

$$
\lim _{m w} \beta_{\mathrm{eff}}^{2}=\beta_{d}^{2}
$$

In the dc limit, the yield stress depends on conductivity differences,

$$
\lim _{m w} \beta_{\mathrm{eff}}^{2}=\beta_{c}^{2} .
$$

The Maxwell-Wagner theory predicts that the dispersion will occur over approximately 2 decades of frequency, and that its shape will depend on the magnitude of $\beta_{d}$ relative to $\beta_{c}$. In barium titanate suspensions, where $\beta_{d} \gg \beta_{c}$, we find that the dynamic yield stress increases substantially with frequency in agreement with the Maxwell-Wagner theory, but that the dispersion occurs over more than 2 decades of frequency.

Although an improvement over the idealized electrostatic polarization model, the Maxwell-Wagner model still does not account for all of the important features of the ER response, one of which is nonlinear conduction [Felici et al. (1994); Foulc et al. (1994)]. For highly polarizable particles, the field strength in the small gap between two closely spaced particles will be much larger than the nominal field strength. The continuous phase conductivity can increase with field strength under these large fields, due to the field-enhanced dissociation of ion pairs [Onsager (1934)]. For highly conductive particles under dc and low frequency ac fields, $\tau_{0} \propto E_{0}^{2} \beta_{c}^{2} \propto E_{0}^{2}\left[\left(\sigma_{p}-\sigma_{c}\right) /\left(\sigma_{p}+2 \sigma_{c}\right)\right]^{2}$. If $\sigma_{c}$ increases with field strength, then the yield stress will increase more slowly with field strength than $E_{0}^{2}$. It has been observed that for large dc fields, the yield stress is proportional to $E_{0}^{3 / 2}$ [Davis and Ginder (1995)]. 


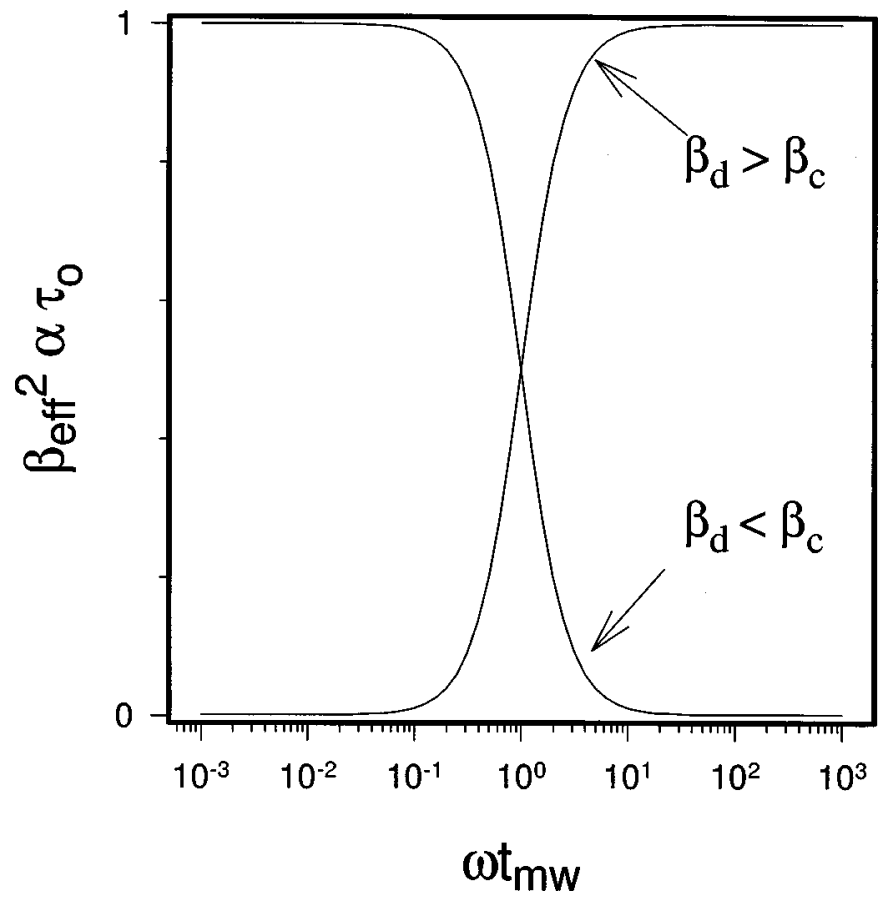

FIG. 1. The effective relative polarizability as a function of $\omega t_{m w}$ for different suspension dielectric properties $\left(\beta_{d}=25.7 \beta_{c}\right.$ and $\left.\beta_{c}=25.7 \beta_{d}\right)$.

Free ions are unable to follow the field at large ac frequencies [Mead and Fuoss (1940); Persoons (1974)], and thus the field enhancement of the conductivity decreases with increasing frequency. Therefore, the field strength dependence of the yield stress should become stronger with increasing frequency as the influence of nonlinear conduction diminishes. Indeed, for the barium titanate suspensions used in this study, the dynamic yield stress is proportional to $E_{0}^{m}$, where $m$ increases with frequency.

The details of sample preparation, as well as rheological, adsorption, and electrical measurements, are described in Sec. II. The results are presented in Sec. III, where the frequency, field strength, and dispersed phase concentration dependence of the ER response of barium titanate suspensions are reported. As expected for large dielectric constant particles, the yield stress of barium titanate suspensions increases with electric field frequency. Nonlinear conduction was examined by measuring the harmonics of the ac current through the suspensions. Current harmonics increased with field strength and decreased with frequency, confirming the existence of frequency dependent nonlinear conduction. Because nonlinear conduction through oil based suspensions was similar to that for suspensions of particles in dry air, we speculate that nonlinear conduction may arise from field-enhanced dissociation of surface groups, rather than from enhanced dissociation of ion pairs within the bulk liquid phase. We also find a greater than linear dependence of the yield stress on dispersed phase concentration. The main results of this work are summarized in Sec. IV.

\section{EXPERIMENT}

\section{A. Sample preparation}

Barium titanate $\left(\mathrm{BaTiO}_{3}\right.$, Aldrich, $99.9 \%$ pure) was ground in a mortar and sifted to obtain particles with diameters in the range 53-90 $\mu \mathrm{m} . \mathrm{BaTiO}_{3}$ has a dielectric constant 
$\epsilon_{p}=2000 \quad\left[\right.$ Ginder and Ceccio (1995)] and an electrical conductivity of $\sigma_{p}$ $\approx 10^{-10} \mathrm{mho} / \mathrm{m}$ [Randall (1997)]. Alumina particles (Aldrich) were also sifted to obtain particle diameters in the same range. Silicone oil (SF96, General Electric, 0.0968 Pa s) was dried for more than 1 week over molecular sieves (Aldrich, 4-8 mesh beads). The sieves were dried for $7 \mathrm{~h}$ under vacuum $(-10 \mathrm{psig})$ at $185^{\circ} \mathrm{C}$ prior to use. Mineral oil (Aldrich) and Brij 30 (Aldrich, $\mathrm{C}_{12} \mathrm{H}_{25}\left(\mathrm{OCH}_{2} \mathrm{CH}_{2}\right)_{4} \mathrm{OH}$; nonionic surfactant) were used as received, as were the following materials needed for the surface treatments described below (each from Aldrich): hexamethyldisilazane (HMDS), hexane, $n$-propanol, octadecanol, and ethanol.

Barium titanate particles were dried at $110^{\circ} \mathrm{C}$ for 2 days and alumina particles at 60 ${ }^{\circ} \mathrm{C}$ for $4 \mathrm{~h}$ prior to suspension preparation. Suspensions were prepared by first weighing a quantity of oil and placing it in a vial. The appropriate amount of particles was added to the vial; for certain suspensions, surfactant was added at this time. The vial was then sealed and vigorously shaken. The suspensions were stored in a sealed desiccator to minimize contact with air. Measurements were performed on suspensions stored for at least $24 \mathrm{~h}$.

Metal oxide particles (e.g., $\mathrm{SiO}_{2}, \mathrm{Al}_{2} \mathrm{O}_{3}, \mathrm{BaTiO}_{3}$ ) exposed to air will possess hydroxyl groups on their surfaces [Hunter (1989)]. Two different standard surface treatments were employed, each with the objective of replacing the surface hydroxyls with hydrophobic molecules on some of the $\mathrm{BaTiO}_{3}$ particles. The treatments, indeed, rendered the particles hydrophobic; untreated particles placed on top of a beaker of water would sink to the bottom, while treated particles would float on the surface.

One treatment was to react surface hydroxyls with HMDS via the reaction [Boehem et al. (1960)],

$$
\mathrm{SiMe}_{3} \cdot \mathrm{NH} \cdot \mathrm{SiMe}_{3}+2-\mathrm{OH} \rightarrow 2-\mathrm{OSiMe}_{3}+\mathrm{NH}_{3} .
$$

Particles were added to a 16 vol \% HMDS/84 vol \% hexane solution and refluxed over a water bath for $2 \mathrm{~h}$. A small amount of $n$-propanol was then added, and the refluxing continued for $2 \mathrm{~h}$ more. The mixture was then vacuum filtered to separate the particles, which were dried as described above.

The other surface treatment was an esterification reaction [Marshall and Zukoski (1990)],

$$
\mathrm{CH}_{3}\left(\mathrm{CH}_{2}\right)_{16} \mathrm{CH}_{2} \mathrm{OH}+-\mathrm{OH} \rightarrow-\mathrm{OCH}_{2}\left(\mathrm{CH}_{2}\right)_{16} \mathrm{CH}_{3}+\mathrm{H}_{2} \mathrm{O} \text {, }
$$

to attach aliphatic chains to the particle surface. Particles were added to an octadecanol/ ethanol solution. The ethanol was boiled off, and the remaining mixture was maintained at $200{ }^{\circ} \mathrm{C}$ for $4 \mathrm{~h}$. The mixture was then filtered and the particles dried as described above.

\section{B. Rheological measurements}

Rheological measurements were made with a Bohlin VOR rheometer using parallel plates, modified for the application of large electric fields [Kim (1996)]. Potential differences were produced by a Stanford Research Systems (model DS345) function generator and amplified by a Trek (model 10/10) amplifier.

Dynamic yield stress measurements consisted of the following steps. The suspension was initially sheared $\left(\dot{\gamma}>10 \mathrm{~s}^{-1}\right)$ at zero field for $1 \mathrm{~min}$ to ensure uniform particle distribution. The desired field was then applied for $1 \mathrm{~min}$ without shear. Shear stresses (averaged over at least 25 periods of the ac electric field) were measured as a function of 


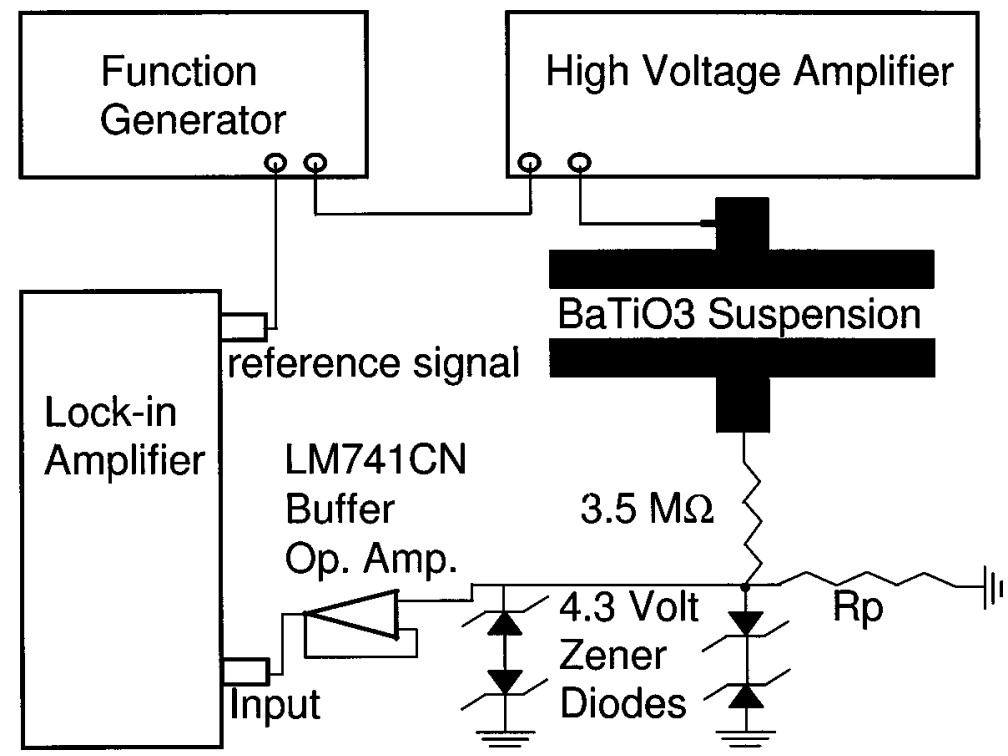

FIG. 2. The circuit design for the harmonic analysis of current through suspensions. The protection circuitry is designed to ensure that no more than $5 \mathrm{~V}$ is sent to the lock-in amplifier. Since the impedance of the suspension decreased with increasing frequency, the applied voltage was adjusted in order to ensure that the voltage drop across the suspension was constant.

decreasing shear rate $\left(0.002 \leqslant \dot{\gamma} \leqslant 3 \mathrm{~s}^{-1}\right)$. The dynamic yield stress was determined by extrapolating the shear stress-shear rate data (over a range of approximately $0.002-0.005$ $\mathrm{s}^{-1}$ ) to zero shear rate.

\section{Electrical measurements}

The dc electrical conductivity of the oils was determined by measuring their resistance to an applied field. Oil was placed between two metal plates and a potential difference of $1 \mathrm{kV}$ applied. A Keithley 5853 picoammeter with the appropriate protection circuitry [Kim (1996)] was placed in series with the sample to measure the steady-state current. The resistance of the sample, $R_{m}$, was calculated from this current. These measurements were repeated for different plate separations, $d$, and the conductivity, $\sigma_{c}$, was determined from

$$
R_{m}=\frac{d}{\sigma_{c} A}+R_{e}
$$

where $A$ is the surface area of the plates and $R_{e}$ is the electrode polarization resistance.

The harmonic analysis of the current through $\mathrm{BaTiO}_{3}$ suspensions was performed using a Stanford Research Systems SR830 lock-in amplifier. The lock-in amplifier, with the appropriate protection circuitry (illustrated in Fig. 2), was placed in series with the sample. Suspensions were placed between the metal rheometer plates, and a potential difference applied. The current harmonics were determined from the potential harmonics measured across the precision resistor, $R_{p}$. 


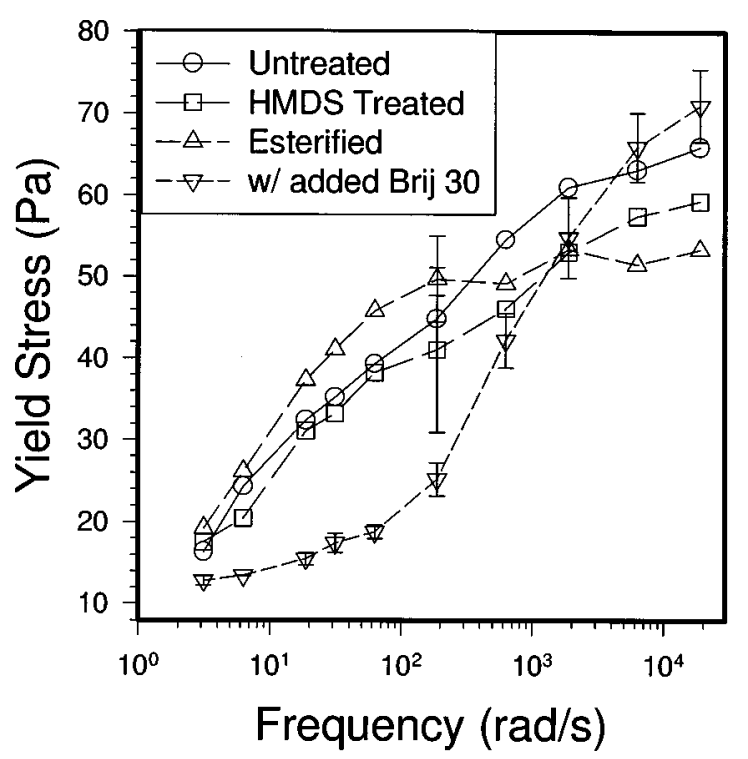

FIG. 3. Yield stress as a function of electric field frequency for $19.3 \mathrm{vol} \% \mathrm{BaTiO}_{3}$ suspensions in silicone oil at $E_{\mathrm{rms}}=750 \mathrm{~V} / \mathrm{mm}$.

\section{Surfactant adsorption}

Brij 30 adsorption was measured by the difference method [Kim (1996)], with the surfactant concentration in solution measured using ultraviolet (UV) absorption. The peak absorption (which occurs between 260 and $300 \mathrm{~nm}$ for Brij 30 solutions [Kim (1996)]) was measured as a function of Brij 30 concentration. Adsorption measurements were performed on a $27 \mathrm{vol} \% \mathrm{BaTiO}_{3}$ suspension. The suspension was mixed thoroughly, and then the particles were allowed to settle for several hours. The absorption of the supernatant was measured, and the amount of surfactant adsorbed determined by difference.

\section{RESULTS AND DISCUSSION}

\section{A. Frequency dependence}

The dynamic yield stress as a function of ac field frequency at $750 \mathrm{~V} / \mathrm{mm}$ for barium titanate/silicone oil suspensions is plotted in Fig. 3. The different symbols represent different surface treatments: untreated barium titanate, HMDS treated particles, esterified particles, and an untreated barium titanate suspension with 0.2 wt \% Brij 30. These suspensions all exhibit Maxwell-Wagner-like behavior. The yield stresses increase with increasing frequency, as expected for suspensions where $\epsilon_{p} \gg \epsilon_{c}$. The suspensions are not very active at low frequencies, but exhibit large yield stresses at high frequencies ( $500 \mathrm{~Pa}$ at $2 \mathrm{kV} / \mathrm{mm})$.

These suspensions show deviations from the Maxwell-Wagner theory. The dispersions are broader than 2 decades in frequency, suggesting that Maxwell-Wagner theory does not accurately describe these systems. This issue is discussed in more detail below.

There is little difference in the frequency dependence of the yield stress between untreated and treated particles, indicating that the surface treatments do not affect the particle polarization. Likewise, changing the continuous phase from silicone oil ( $\sigma_{c}$ $\left.\approx 10^{-11} \mathrm{mho} / \mathrm{m}\right)$ to mineral oil $\left(\sigma_{c} \approx 3 \times 10^{-13} \mathrm{mho} / \mathrm{m}\right)$ does not alter the frequency 


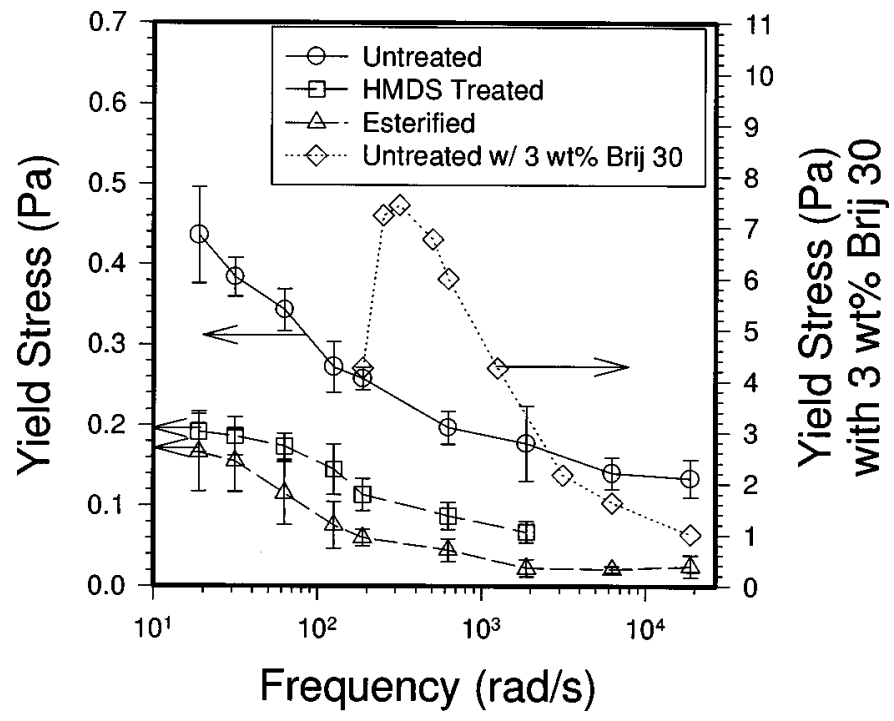

FIG. 4. Yield stress as a function of frequency for both untreated and treated $20 \mathrm{vol} \%$ alumina/silicone oil suspensions at $E_{\mathrm{rms}}=1 \mathrm{kV} / \mathrm{mm}$. Also shown are results for a $20 \mathrm{vol} \%$ untreated alumina/silicone oil suspension with 3 wt $\%$ Brij 30 at $E_{\mathrm{rms}}=1 \mathrm{kV} / \mathrm{mm}[\mathrm{Kim}(1996)]$.

response, implying that the continuous phase conductivity does not control particle polarization. Adding surfactant, however, increases the apparent characteristic frequency. According to the Maxwell-Wagner theory, an increase in the characteristic frequency corresponds to a decrease in $t_{m w}$, which for these suspensions $\left(\epsilon_{p} \gg \epsilon_{c}\right)$ is approximately

$$
t_{m w} \cong \epsilon_{0} \frac{\epsilon_{p}}{\sigma_{p}+\frac{2 \lambda^{s}}{a}+2 \sigma_{c}} .
$$

Therefore, the addition of Brij 30 increases $\sigma_{p}, \sigma_{c}$, or $\lambda_{s}$.

Kim (1996) showed that the ER response of alumina/silicone oil suspensions strongly depends on the magnitude of the surface conductivity. The addition of Brij 30 was reported to dramatically alter the ER response by mobilizing charge carriers near the solid/liquid interface. Absorption measurements indicate that at $0.2 \mathrm{wt} \%$, Brij 30 strongly adsorbs to barium titanate particles in $19.3 \mathrm{vol} \%$ suspensions. The change in the dispersion upon adding Brij 30 to barium titanate suspensions is thus apparently due to an increase in the surface conductivity.

Although the different surface treatments have no effect on the dispersion of barium titanate suspensions, Fig. 4 illustrates that the surface treatments do affect the ER response of 20 volume\% alumina/silicone oil suspensions at $1 \mathrm{kV} / \mathrm{mm}$. Again, MaxwellWagner qualitatively describes the ER response; the yield stress decreases with frequency, as expected since $\beta_{c}>\beta_{d}$. Consistent with a decrease in the number of mobile surface protons (i.e., surface hydroxyl groups), the surface treatments significantly alter the ER response of alumina suspensions, decreasing the yield stress by more than $50 \%$.

The different effects of surface treatments on the ER response of barium titanate and alumina suspensions may be attributed to the relative importance of surface conductivity 


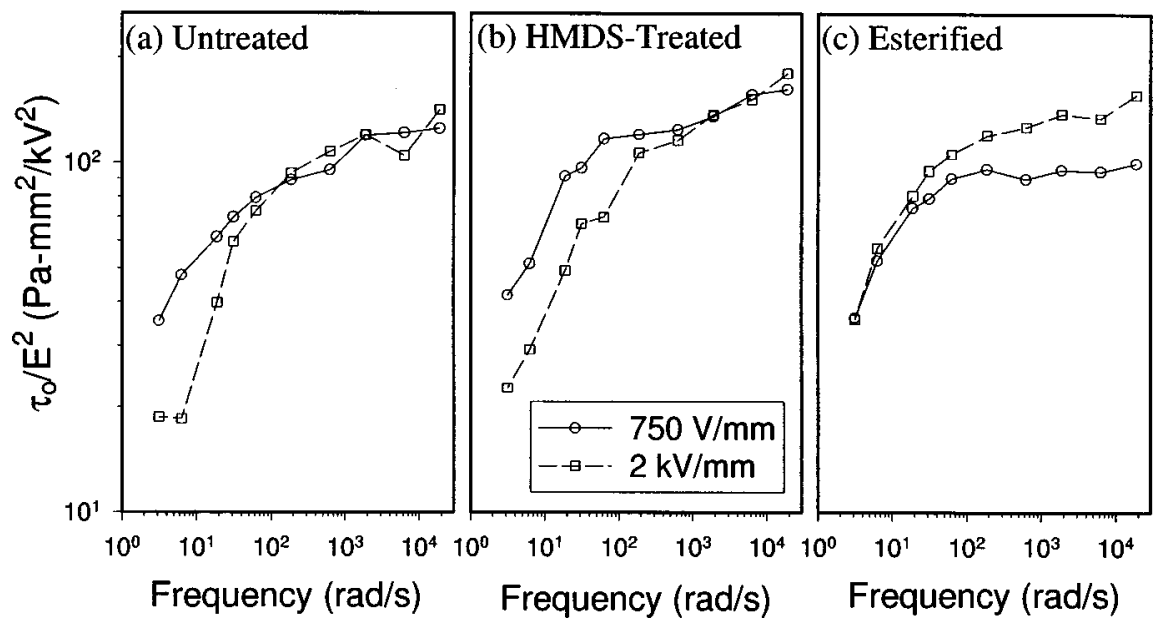

FIG. 5. $\tau_{0} / E_{\mathrm{rms}}^{2}$ as a function of frequency for $19.3 \mathrm{vol} \%$ (a) untreated, (b) HMDS treated, and (c) esterified $\mathrm{BaTiO}_{3}$ suspensions in silicone oil at $2 \mathrm{kV} / \mathrm{mm}$ and $750 \mathrm{~V} / \mathrm{mm}$.

in the two types of suspensions. The surface treatments replaced surface hydroxyl groups with siloxane groups or aliphatic chains, rendering the surfaces hydrophobic. The removal of potential charge carriers (e.g., protons) should decrease the surface conductance. Alumina/silicone oil ER suspensions are very sensitive to the level of surface conductivity, so the observed impact of the surface treatments is expected. The decreased surface conductivity reduces interfacial polarization, leading to a weaker ER response.

We believe that the surface treatments also reduce the surface conductivity of barium titanate. Since the bulk conductivity $\left(\sigma_{p} \approx 10^{-10} \mathrm{mho} / \mathrm{m}\right)$ is relatively large, however, decreasing the surface conductivity has little impact on the interfacial polarization (i.e., $\sigma_{p} \gg 2 \lambda_{s} / a$ for both treated and untreated $\mathrm{BaTiO}_{3}$ suspensions). Similarly, changing the continuous phase does not alter the dispersion; the bulk particle conductivity is at least an order of magnitude greater than the conductivity of either oil, so the change has no effect on the polarization. The addition of Brij 30, however, increases the surface conductivity, such that its contribution to the net interfacial polarization is sufficient to increase the characteristic frequency.

We therefore conclude that the dielectric relaxation (as observed in the ER frequency response) of barium titanate/insulating oil suspensions without surfactant is determined by the bulk dielectric properties of the dispersed phase. In the Appendix, we show that we obtain the same conclusion when we employ a different model for the particle morphology - a particle core surrounded by a thin leaky dielectric layer, as opposed to a particle with a surface conductivity, $\lambda_{s}$.

\section{B. Field strength dependence}

\section{Rheological analysis}

The frequency dependence of the yield stress depends on the electric field strength for barium titanate suspensions. According to the Maxwell-Wagner theory, the dynamic yield stress should be proportional to $E_{\mathrm{rms}}^{2}$, and thus the data at different field strengths plotted as $\tau_{0} / E_{\mathrm{rms}}^{2}$ vs $\omega$ should collapse onto a single curve. $\tau_{0} / E_{\mathrm{rms}}^{2}$ is plotted as a function of frequency for two different field strengths, for untreated and both surfacetreated barium titanate suspensions in Fig. 5. The data do not collapse onto a single curve. 
For untreated and HMDS treated particles at low frequencies, the yield stress is proportional to $E_{\mathrm{rms}}^{m}$, with $m<2$, consistent with field-dependent conduction in the interparticle gap [Foulc et al. (1994)]. The data do superimpose with increasing frequency, suggesting that the conductivity has both a field strength and frequency dependence, where

$$
\begin{aligned}
& \left(\frac{\partial \sigma_{c}}{\partial E}\right)_{\omega}>0, \\
& \left(\frac{\partial \sigma_{c}}{\partial \omega}\right)_{E}<0 .
\end{aligned}
$$

The dynamic yield stress as a function of field strength at large frequencies $(2000 \pi$ $\mathrm{rad} / \mathrm{s}$ ) was proportional to $E^{2}$ down to fields as small as $100 \mathrm{~V} / \mathrm{mm}$. Therefore, despite observing a slight degree of flocculation, it is apparent that the colloidal contribution to the yield stress is insignificant compared to the electrostatic contribution.

In the esterified barium titanate suspensions, the data superimpose at low frequencies, but the yield stress is proportional to $E_{\mathrm{rms}}^{m}$, with $m>2$ at higher frequencies. We believe that as the field strength increases, the grafted $C_{18}$ chains compress due to the increased electrostatic interparticle force. This can give rise to an interparticle force that increases more rapidly than $E^{2}$. The interparticle force, nominally scaling with $E^{2}$, is a monotonically decreasing function of separation. Thus if the separation decreases with field strength, the observed force will increase more rapidly than $E^{2}$. The yield stress will then vary as $E^{m}$, with $m>2$. Since $m$ increases with frequency, it appears that nonlinear conduction influences the rheological response in a manner similar to that in the other suspensions.

The Maxwell-Wagner theory predicts that the dispersion will occur over approximately 2 decades of frequency, but it is apparent from Figs. 3 and 5 that the dispersion of barium titanate/silicone oil suspensions covers significantly more than 2 decades. The dispersion also becomes broader with increasing field strength. Both of these trends are consistent with frequency-dependent field-enhanced conduction in the interparticle gap.

\section{Harmonic analysis}

The current density in the suspension, $j(t)$, can be expressed as a multiple integral expansion arising from the Boltzmann superposition theory [Ward (1971)],

$$
\begin{aligned}
j(t)= & \int_{-\infty}^{t} \sigma_{1}(t-\tau) \frac{\partial E(\tau)}{\partial \tau} \partial \tau+\int_{-\infty}^{t} \int_{-\infty}^{t} \sigma_{2}\left(t-\tau_{1}, t-\tau_{2}\right) \frac{\partial E\left(\tau_{1}\right)}{\partial \tau_{1}} \frac{\partial E\left(\tau_{2}\right)}{\partial \tau_{2}} \partial \tau_{1} \partial \tau_{2} \\
& +\cdots+\int_{-\infty}^{t} \cdots \int_{-\infty}^{t} \sigma_{N}\left(t-\tau_{1}, \cdots, t-\tau_{N}\right) \frac{\partial E\left(\tau_{1}\right)}{\partial \tau_{1}} \cdots \frac{\partial E\left(\tau_{N}\right)}{\partial \tau_{N}} \partial \tau_{1} \cdots \partial \tau_{N},
\end{aligned}
$$

where the $\sigma_{i}$ are the conductivity memory functions. If the electric field is sinusoidal, $E(t)=E_{0} \sin \omega t$, the above expression gives the current density expressed as a series of odd harmonics,

$$
j(t)=f_{1}\left(E_{0}\right) \sin \omega t+f_{3}\left(E_{0}\right) \sin 3 \omega t+f_{5}\left(E_{0}\right) \sin 5 \omega t+\ldots .
$$

An increase in the current harmonics' magnitudes $\left[f_{k}\left(E_{0}\right)\right]$ with field strength indicates an increasing suspension conductivity with field strength.

The harmonics of the current passing through barium titanate suspensions at different field strengths (applied frequency of $60 \pi \mathrm{rad} / \mathrm{s}$ ) are illustrated in Fig. 6. The existence of 

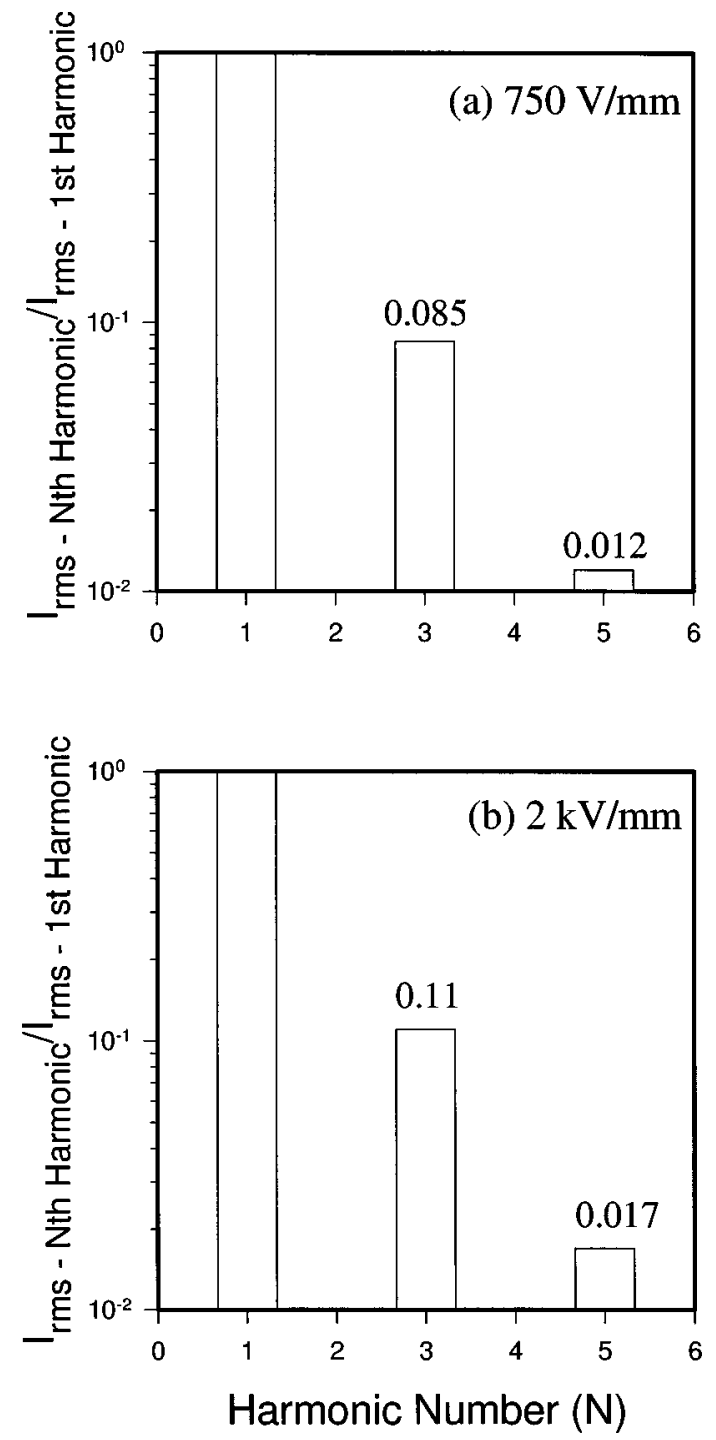

FIG. 6. Harmonics of current through 19.3 vol \% untreated barium titanate/silicone oil suspensions at (a) 750 $\mathrm{V} / \mathrm{mm}$ and (b) $2 \mathrm{kV} / \mathrm{mm}$ (applied frequency of $60 \pi \mathrm{rad} / \mathrm{s}$ ).

odd harmonics verifies that the suspension conductivity is field dependent. The harmonics (relative to the fundamental harmonic) increase with field strength, indicating that the apparent suspension conductivity increases with field strength.

The ratio of the third to the first current harmonics is plotted as a function of field frequency for different field strengths in Fig. 7. The harmonics decrease with frequency, indicating a reduction in the field enhancement of conduction with increasing frequency. The field strength and frequency dependence of the phase of the current (relative to the field) is consistent with a decrease in suspension resistance with increasing field strength.

It has been suggested that the frequency dependence of the field enhancement of the continuous phase conductivity is [Persoons (1974)] 


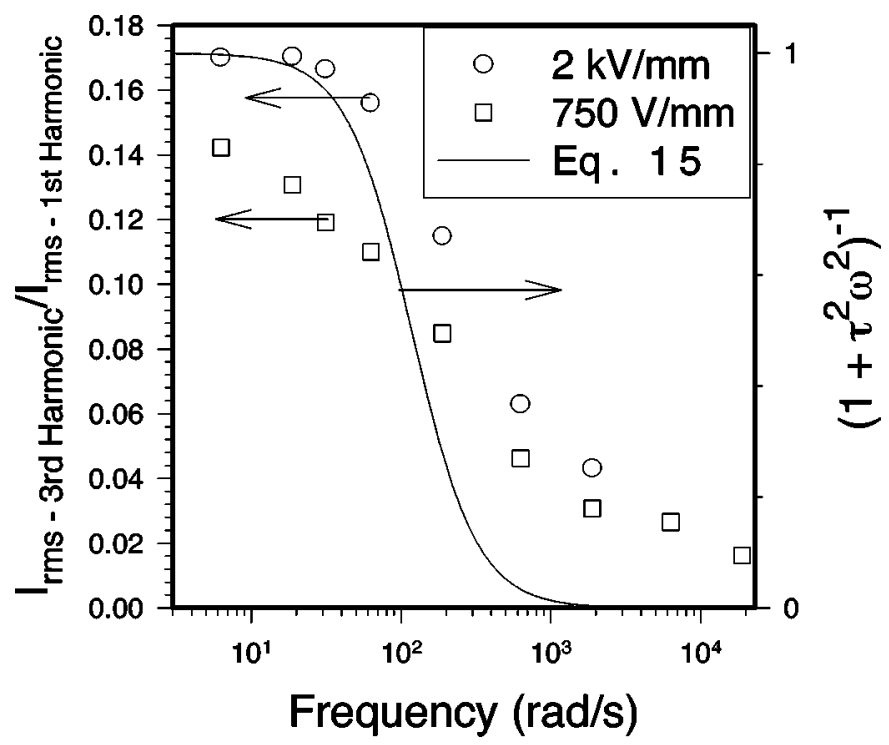

FIG. 7. The third current harmonics (relative to the first current harmonics) through a $\mathrm{BaTiO}_{3} / \mathrm{silicone}$ oil suspension as a function of frequency at $750 \mathrm{~V} / \mathrm{mm}$ and $2 \mathrm{kV} / \mathrm{mm}$. Also plotted is the normalized oil conductivity enhancement [as suggested by Persoons (1974)] for a relaxation time of $\tau=0.0085 \mathrm{~s}$ (chosen to fall in approximately the same frequency range as the experimental data).

$$
\Delta \sigma_{c}(E, \omega)=\frac{\Delta \sigma_{c}^{\mathrm{dc}}(E)}{1+\omega^{2} \tau^{2}},
$$

where $\Delta \sigma_{c}^{\mathrm{dc}}(E)$ is the dc field enhancement of the fluid conductivity predicted by Onsager (1934), $\omega$ is the electric field frequency, and $\tau$ is a characteristic relaxation time. As shown in Fig. 7, the frequency dependence of this function qualitatively follows the same trend as the measured third harmonics, although the agreement is far from quantitative.

The third current harmonic of a barium titanate/nitrogen enriched air suspension is plotted as a function of frequency at $750 \mathrm{~V} / \mathrm{mm}$ in Fig. 8. In these experiments, dry $\mathrm{BaTiO}_{3}$ particles are placed directly on the bottom plate. The top plate is then lowered until it contacts the particles. The potential difference is applied; the top plate is then raised to a desired height and the potential difference is adjusted to achieve the desired field strength. The entire rheometer is placed in a chamber flushed continuously with nitrogen at 6 psig.

The frequency behavior and magnitude of the harmonics in air are very similar to those of silicone oil suspensions. Since the number density of ion pairs should be much smaller in dry air than in silicone oil, this result suggests that the observed nonlinear conduction does not arise from field-enhanced dissociation of ion pairs within the continuous phase. We speculate that a more likely explanation is that the nonlinear conduction is produced by field-enhanced dissociation of chemical groups on the particle surfaces, rather than in the bulk liquid phase. Confirming this hypothesis requires more information. This distinction may be important, for example, in engineering suspensions with tailored conduction behavior. 


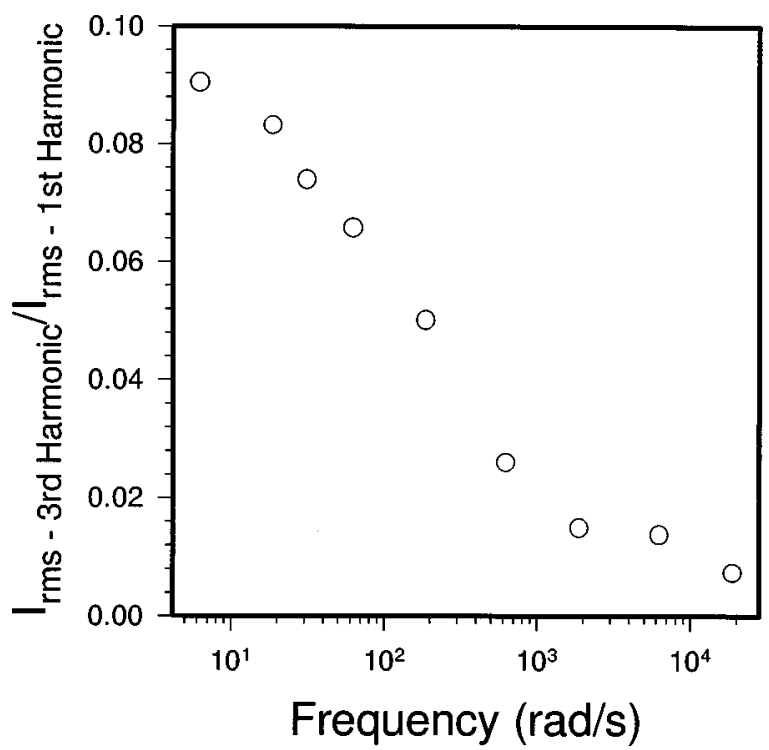

FIG. 8. The third harmonic of the current through a $\mathrm{BaTiO}_{3} /$ nitrogen enriched air suspension at $750 \mathrm{~V} / \mathrm{mm}$ as a function of frequency.

\section{Suspension concentration dependence}

The dynamic yield stress is plotted as a function of particle concentration for untreated barium titanate/silicone oil suspensions at $750 \mathrm{~V} / \mathrm{mm}$ and various frequencies in Fig. 9. The yield stress increases faster than linearly with concentration, in contrast to previously reported theoretical predictions [Klingenberg and Zukoski(1990); Bonnecaze and Brady (1992)].

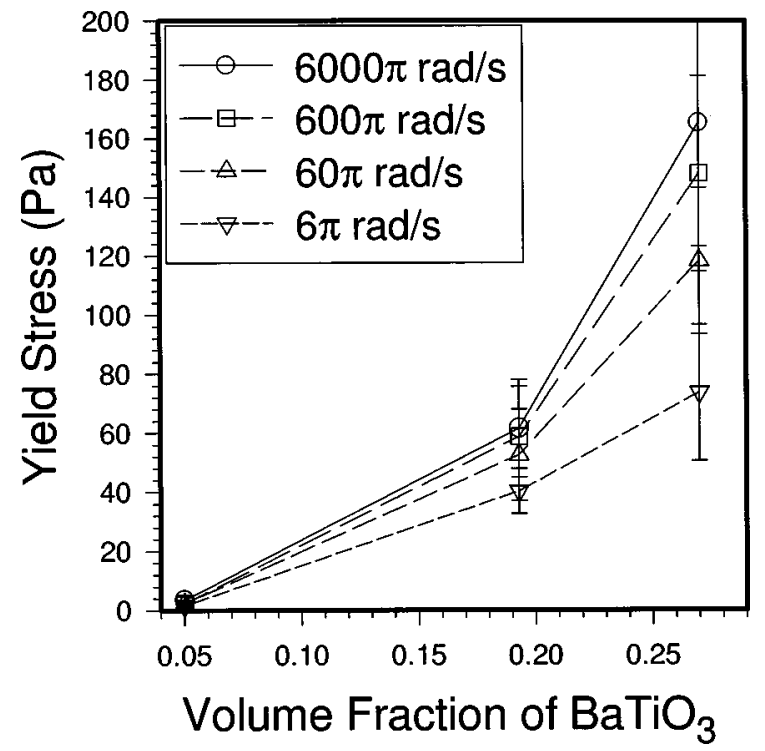

FIG. 9. Dynamic yield stress as a function of particle concentration for $\mathrm{BaTiO}_{3}$ in silicone oil at $750 \mathrm{~V} / \mathrm{mm}$ and various frequencies. 
A nonlinear concentration dependence may potentially arise from a variety of mechanisms: multibody electrostatic interactions [Bonnecaze and Brady (1992)], changes in microstructure with concentration [Klingenberg et al. (1991)], or the influence of gravity. The importance of buoyancy on particle dynamics can be assessed by considering the ratio of viscous and polarization forces to gravitational forces. Gast and Zukoski (1989) reported that viscous forces in ER suspensions scale as $\eta_{c} a^{2} \dot{\gamma}$ and polarization forces scale as $\epsilon_{0} \epsilon_{c} a^{2} \beta_{\mathrm{eff}}^{2} E_{\mathrm{rms}}^{2}$. Gravitational forces scale as $a^{3}\left(\rho_{p}-\rho_{c}\right) g$, leading to the force ratios

$$
\begin{gathered}
\frac{\text { Viscous Forces }}{\text { Gravitational Forces }} \sim \frac{\eta_{c} \dot{\gamma}}{a\left(\rho_{p}-\rho_{c}\right) g} \approx 0.017, \\
\frac{\text { Polarization Forces }}{\text { Gravitational Forces }} \sim \frac{\epsilon_{0} \epsilon_{c} \beta_{\mathrm{eff}}^{2} E_{\mathrm{rms}}^{2}}{a\left(\rho_{p}-\rho_{c}\right) g} \approx 3 .
\end{gathered}
$$

(Here we have used the following parameter values: $\eta_{c}=0.1 \mathrm{Pas}, \dot{\gamma}=1 \mathrm{~s}^{-1}, a$ $=3.5 \times 10^{-5} \mathrm{~m}, \rho_{p}-\rho_{c}=5.1 \times 10^{3} \mathrm{~kg} / \mathrm{m}^{3}, g=9.8 \mathrm{~m} / \mathrm{s}^{2}, \epsilon_{c}=2.7, \beta_{\text {eff }}=0.9$, and $E_{\mathrm{rms}}=10^{6} \mathrm{~V} / \mathrm{m}$.) Thus for barium titanate suspensions, where particle densities are approximately six times that of the oil densities, gravitational forces may be strong enough to influence particle dynamics and therefore the suspension structure (especially for sufficiently small shear rates and electric field strengths). Whether or not gravitational forces can produce the nonlinear volume fraction dependence of the yield stress depicted in Fig. 9 remains to be determined.

Although all of the trends reported throughout this paper were the same for each sample, the yield stress magnitudes varied, most likely as a result of sampling inconsistency. After mixing a suspension in a vial, some degree of sedimentation occurred before a sample could be extracted. Thus sample concentrations were not equal to the vial concentrations, and varied among samples. This variability in suspension concentration, coupled with the sensitivity of the yield stress to dispersed phase concentration, most likely caused the variability in the data.

\section{CONCLUSION}

Barium titanate suspensions show a substantial electrorheological response, exhibiting a dynamic yield stress of approximately $500 \mathrm{~Pa}$ upon the application of an electric field of $2 \mathrm{kV} / \mathrm{mm}$ and $1 \mathrm{kHz}$ (19.3 vol \% suspensions). The responses qualitatively follow predictions from the Maxwell-Wagner theory for high permittivity dispersed phases, with the yield stress increasing with ac field frequency.

The bulk conductivity of barium titanate controls the dielectric relaxation in suspensions without surfactant. Decreasing the surface conductivity with surface treatments and decreasing the continuous phase conductivity by changing the suspending medium from silicone to mineral oil had no effect on the dispersion. The addition of a small amount of adsorbed Brij 30, however, changed the dispersion in a manner consistent with a substantial increase in surface conductivity.

The ER response of these suspensions is very sensitive to the particle concentration. The dynamic yield stress shows a greater than linear dependence on particle concentration. This nonlinear dependence may arise from the influence of buoyancy.

Frequency dependent nonlinear conduction affects the ER response of barium titanate suspensions. At fixed frequency, the dynamic yield stress is proportional to $E^{m}$, where $m$ increases with frequency, consistent with frequency-dependent nonlinear conduction. The 
dispersion is broader than that predicted by the Maxwell-Wagner theory, and broadens with increasing field strength. The magnitude of the suspension current harmonics increases with field strength and decreases with frequency, These observations further illustrate the frequency dependence of the nonlinear conduction. Changing the suspending medium from silicone oil to dry air does not significantly alter the current harmonics, suggesting that nonlinear conduction arises from field-enhanced dissociation of particle surface groups, and not from field-enhanced ion-pair dissociation in the continuous phase.

Nonlinear conduction poses a severe hindrance to the development of suitable ER fluids, limiting the sensitivity of the yield stress to the electric field strength. Furthermore, the substantial increase in suspension conductivity with field strength increases the power required, rendering such suspensions unsuitable for many proposed applications. Our results suggest that efforts to combat nonlinear conduction should focus on particle surfaces rather than the suspending medium, since it is the surface properties that apparently determine the degree of nonlinear conduction.

\section{ACKNOWLEDGMENTS}

The authors thank S. H. Langer, Y. Shkel, M. Parthasarathy and I. Shkel for helpful discussions. This work was supported by the National Science Foundation under Grant No. CTS-9401293.

\section{APPENDIX}

The main conclusions of Section III A were:

(i) Surface treatments reduce the surface conductivity, but do not alter the dispersion for barium titanate suspensions;

(ii) Adding surfactant increases the surface conductivity, and does shift the dispersion to higher frequencies;

(iii) The frequency dependence of barium titanate/insulating oil suspensions without surfactant is controlled by the bulk particle conductivity.

These conclusions were obtained by considering the particles as leaky dielectrics with infinitesimally thin conducting layers on their surfaces. In this Appendix, we show that the same conclusions can be obtained for a different model of the particle morphology.

We model the composite particle as a leaky dielectric core (radius $a$, dielectric constant $\epsilon_{p}$, conductivity $\sigma_{p}$ ) surrounded by a leaky dielectric shell (thickness $\delta$, dielectric constant $\epsilon_{s}$, conductivity $\sigma_{s}$ ) as illustrated in Fig. 10. The core represents the bulk barium titanate phase. The shell represents the interfacial region, which includes any adsorbed and grafted species, as well as any near-surface layer of the barium titanate that may differ in structure from the bulk phase. This thin shell region replaces that represented by the surface conductivity, $\lambda^{s}$, in Sec. III A. The particles are immersed in a leaky dielectric continuous phase (dielectric constant $\epsilon_{c}$, conductivity $\sigma_{c}$ ).

The polarization of such a composite particle in an ac electric field can be obtained by solving for the complex electrostatic potential [Davis (1993); Tang and Conrad (1996); Parthasarathy and Klingenberg (1996)]. The resulting effective relative polarizability of the composite particle is 


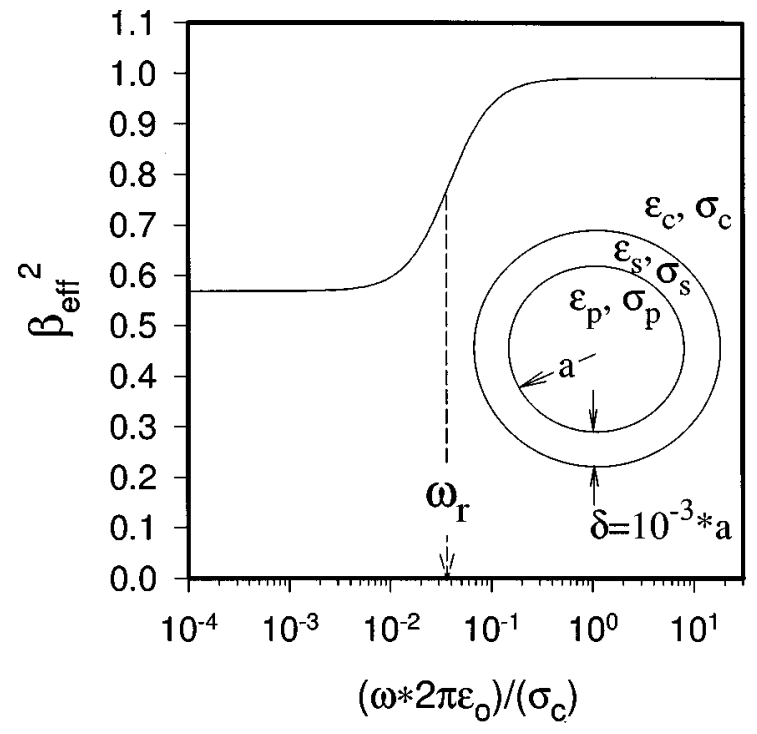

FIG. 10. The effective relative polarizability as a function of $\omega /\left(\sigma_{c} / 2 \pi \epsilon_{0}\right) . \epsilon_{p}=2 \times 10^{3}, \sigma_{p}=1$ $\times 10^{-10} \mathrm{mho} / \mathrm{m}, \epsilon_{c}=2.75, \sigma_{c}=1 \times 10^{-11} \mathrm{mho} / \mathrm{m}, \epsilon_{s}=1 \times 10^{1}, \sigma_{s}=1 \times 10^{-9} \mathrm{mho} / \mathrm{m}$, and $\delta / a=1$ $\times 10^{-3}$.

$$
\beta_{\mathrm{eff}}^{2}=\left|\frac{\frac{\epsilon_{p}^{*}-\epsilon_{s}^{*}}{\epsilon_{p}^{*}+2 \epsilon_{s}^{*}} \frac{2 \epsilon_{s}^{*}+\epsilon_{c}^{*}}{\epsilon_{s}^{*}-\epsilon_{c}^{*}}\left(\frac{R}{R+\delta}\right)^{3}+1}{2 \frac{\epsilon_{p}^{*}-\epsilon_{s}^{*}}{\epsilon_{p}^{*}+2 \epsilon_{s}^{*}}\left(\frac{R}{R+\delta}\right)^{3}+\frac{\epsilon_{s}^{*}+2 \epsilon_{c}^{*}}{\epsilon_{s}^{*}-\epsilon_{c}^{*}}}\right|^{2},
$$

where $\epsilon_{k}^{*}=\epsilon_{k}-j \sigma_{k} / \epsilon_{0} \omega$ is the complex dielectric constant of phase $k$, and $j$ $=\sqrt{-1}$.

The effective relative polarizability is plotted as a function of frequency (scaled by $\left.\sigma_{c} / 2 \pi \epsilon_{0}\right)$ in Fig. 10, for the parameter values:

$$
\begin{array}{ll}
\epsilon_{p}=2 \times 10^{3}, & \sigma_{p}=1 \times 10^{-10} \mathrm{mho} / \mathrm{m}, \\
\epsilon_{c}=2.75, & \sigma_{c}=1 \times 10^{-11} \mathrm{mho} / \mathrm{m}, \\
\epsilon_{s}=1 \times 10^{1}, & \sigma_{s}=1 \times 10^{-9} \mathrm{mho} / \mathrm{m}, \\
\delta / a=1 \times 10^{-3} . &
\end{array}
$$

The parameters values for the particle core and continuous phase correspond to the values for barium titanate and silicone oil, respectively. $\epsilon_{s}$ was chosen to lie between $\epsilon_{p}$ and $\epsilon_{c}$, but otherwise chosen arbitrarily; varying its value within this range does not significantly affect $\beta_{\text {eff }}^{2}(\omega)$. We expect $\sigma_{s} \gtrless \sigma_{p}$ since this region contains barium titanate plus any mobile charge carriers at the interface. The influence of $\sigma_{s}$ on the dispersion of $\beta_{\text {eff }}^{2}(\omega)$ is discussed below.

The dispersion of $\beta_{\mathrm{eff}}^{2}(\omega)$ for these parameters closely resembles classical MaxwellWagner behavior for high dielectric constant particles. To investigate the impact of 


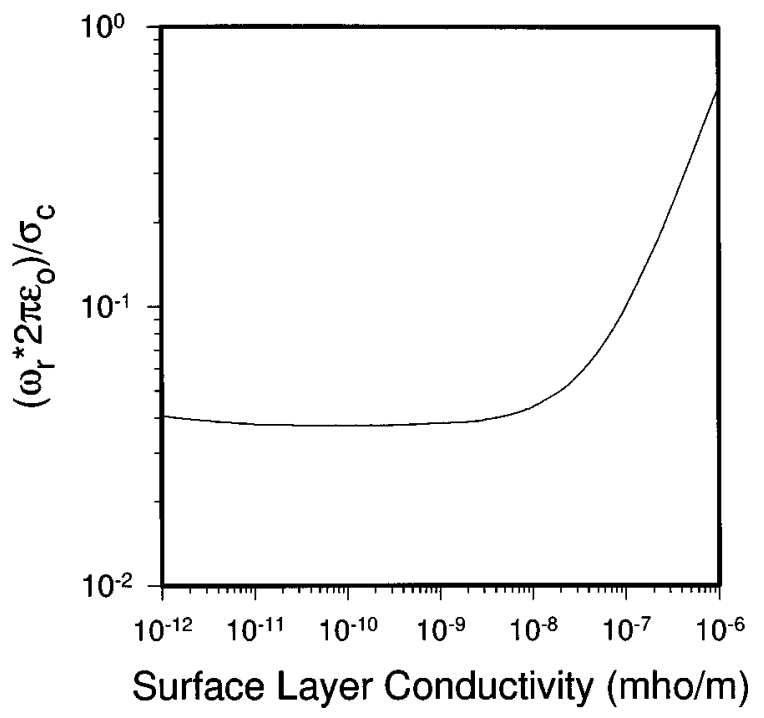

FIG. 11. Characteristic relaxation frequency as a function of $\sigma_{s} . \epsilon_{p}=2 \times 10^{3}, \sigma_{p}=1 \times 10^{-10} \mathrm{mho} / \mathrm{m}$, $\epsilon_{c}=2.75, \sigma_{c}=1 \times 10^{-11} \mathrm{mho} / \mathrm{m}, \epsilon_{s}=1 \times 10^{1}$, and $\delta / a=1 \times 10^{-3}$.

various chemical modifications on the dispersion, represented as variations of such parameters as $\sigma_{c}$ and $\sigma_{s}$, we define a characteristic relaxation frequency, $\omega_{r}$,

$$
\beta_{\mathrm{eff}}^{2}\left(\omega_{r}\right) \equiv \frac{1}{2}\left[\beta_{\mathrm{eff}}^{2}(\omega \rightarrow 0)+\beta_{\mathrm{eff}}^{2}(\omega \rightarrow \infty)\right]
$$

as illustrated in Fig. 10.

Decreasing $\sigma_{c}$ by a factor of 33 (to that of mineral oil) decreases $\omega_{r}$ by only a factor of 1.4. This change is quite small compared to the experimental uncertainty (see Fig. 3), and thus we conclude, as before, that the continuous phase conductivity does not control the dispersion for these suspensions.

The characteristic relaxation frequency is plotted as a function of $\sigma_{s}$ in Fig. 11. The characteristic frequency is essentially independent of $\sigma_{s}$ for values down to at least two orders of magnitude smaller than $\sigma_{p}\left(=1 \times 10^{-10} \mathrm{mho} / \mathrm{m}\right)$. The characteristic frequency does, however, begin to increase when $\sigma_{s}$ is increased to more than an order of magnitude larger than $\sigma_{p}$. We therefore conclude, as before, that although the surface treatment reactions decrease the shell (surface) conductivity, this has no impact on the dispersion of the yield stress. Increasing the shell (surface) conductivity by adding surfactant, however, can shift the characteristic frequency to larger values, as observed experimentally.

The conclusion that the dielectric relaxation of barium titanate/insulating oil suspensions without surfactant is determined by the bulk dielectric properties of the dispersed phase is therefore supported by the core/shell model for the particle dielectric properties.

\section{References}

Anderson, R. A., "Effects of finite conductivity in electrorheological fluids," in Proceedings of the 3rd International Conference of Electrorheological Fluids, edited by R. Tao (World Scientific, Singapore, 1992), pp. 81-90.

Block, H. and J. P. Kelley, “Electro-rheology,” J. Phys. D 21, 1661-1677 (1988). 
Boehem, J., S. H. Langer, R. H. Perrett, and J. H. Purnell, "A study of the adsorptive properties of firebrick in relation to its use as a solid support in gas-liquid chromatography," J. Chem. Soc. 494, 2444-2451 (1960).

Bonnecaze, R. T. and J. F. Brady, "Yield stresses in electrorheological fluids," J. Rheol. 36, 73-115 (1992).

Davis, L. C., "Finite-element analysis of particle-particle forces in electrorheological fluids," Appl. Phys. Lett. 60, 319-321 (1992a).

Davis, L. C., "Polarization forces and conductivity effects in electrorheological fluids," J. Appl. Phys. 72, 1334-1340 (1992b).

Davis, L. C., "The metal-particle/insulating oil system: an ideal electrorheological fluid," J. Appl. Phys. 73, 680-683 (1993).

Davis, L. C. and J. M. Ginder, "Electrostatic forces in electrorheological fluids,” Progress in Electrorheology, edited by K. O. Havelka and F. E. Filisko (Plenum, New York, 1995), pp. 107-114.

Deinega, Y. F. and G. V. Vinogradov, "Electric fields in the rheology of dispersed systems," Rheol. Acta 23, 636-651 (1984).

Felici, N., J. N. Foulc, and P. Atten, "A conduction model of electrorheological effect," in Proceedings of the 4th International Conference of Electrorheological Fluids, edited by R. Tao (World Scientific, Singapore 1994), pp. 139-152.

Foulc, J. N., P. Atten, and N. Felici, "Macroscopic model of interaction between particles in an electrorheological fluid," J. Electrostat. 33, 103-112 (1994).

Gast, A. P. and C. F. Zukoski, "Electrorheological fluids as colloidal suspensions," Adv. Colloid Interface Sci. 30, 153-202 (1989).

Ginder, J. M. and S. L. Ceccio, "Transient stresses in electrorheological fluids: Role of particle polarization dynamics," J. Rheol. 39, 211-224 (1995).

Hartsock, D. L., R. F. Novak, and G. J. Chaundy, “ER fluid requirements for automotive devices,' J. Rheol. 35, 1305-1326 (1991).

Hunter, R. J., Foundations of Colloid Science (Clarendon, Oxford, 1989), Vol. 1.

Jordan, T. C. and M. T. Shaw, "Electrorheology," IEEE Trans. Electron. Insul. 24, 849-878 (1989).

Kim, Y. D., Ph.D. thesis, University of Wisconsin, Madison, WI, 1996.

Klingenberg, D. J., D. Dierking, and C. F. Zukoski, “'Stress-transfer mechanisms in electrorheological suspensions," J. Chem. Soc. Faraday Trans. 87, 425-430 (1991).

Klingenberg, D. J. and C. F. Zukoski, "Studies on the steady shear behavior of electrorheological suspensions," Langmuir 6, 15-24 (1990).

Marshall, L. and C. F. Zukoski IV, "Experimental studies on the rheology of hard-sphere suspensions near the glass transition," J. Phys. Chem. 94, 1164-1171 (1990).

Mead, D. J. and R. M. Fuoss, "Dependence of conductance on field strength II. Tetrabutylammonium bromide in diphenyl ether at 50',' J. Am. Chem. Soc. 62, 1720-1723 (1940).

O'Konski, C. T., "Electric properties of macromolecules V. Theory of ionic polarization in polyelectrolytes," J. Phys. Chem. 64, 605-619 (1960).

Onsager, L., "Deviations from Ohm's law in weak electrolytes," J. Chem. Phys. 2, 599-615 (1934).

Parthasarathy, M. and D. J. Klingenberg, "Electrorheology: Mechanisms and models," Mater. Sci. Eng. R17, 57-103 (1996).

Persoons, A. P., "Field dissociation effect and chemical relaxation in electrolyte solutions of low polarity," J. Phys. Chem. 78, 1210-1217 (1974).

Randall, C. (private communication, 1997).

Tang, X. and H. Conrad, "The role of dielectric relaxation in transient electrorheological response," J. Appl. Phys. 80, 5240-5247 (1996).

Ward, I. M. Mechanical Properties of Solid Polymers (Wiley, New York, 1971), pp. 214-218.

Weiss, K. D., J. D. Carlson, and J. P. Coulter, “Material aspects of electrorheological systems,'” J. Intell. Mater. Syst. Struct. 4, 13-34 (1993).

Winslow, W. M., “'Induced fibration of suspensions," J. Appl. Phys. 20, 1137-1140 (1949). 